Revue internationale P.M.E.

Économie et gestion de la petite et moyenne entreprise

\title{
PME, gestion internationale et systèmes d'information marketing : au-delà des évidences technologiques
}

\section{Martine Boutary}

Volume 13, numéro 3-4, 2000

URI : https://id.erudit.org/iderudit/1008679ar

DOI : https://doi.org/10.7202/1008679ar

Aller au sommaire du numéro

Éditeur(s)

Presses de l’Université du Québec

ISSN

0776-5436 (imprimé)

1918-9699 (numérique)

Découvrir la revue

\section{Citer cet article}

Boutary, M. (2000). PME, gestion internationale et systèmes d'information marketing : au-delà des évidences technologiques. Revue internationale P.M.E., 13(3-4), 9-36. https://doi.org/10.7202/1008679ar

\section{Résumé de l'article}

La connaissance des marchés a souvent été mise en évidence comme étant indispensable à la réussite internationale. Les structures exportatrices de taille moyenne sont particulièrement sensibles à ce problème : leur besoin informationnel est rendu important par des marchés géographiquement dispersés, alors que leurs systèmes d'information sont le plus souvent peu formalisés, peu sophistiqués et vite surchargés. La tentation d’un discours normatif est alors grande et de nombreux conseils de gestion de Information, soutenus par les informaticiens, sont mis en place.

La problématique abordée est celle d'une performance sur le marché international soumise à des conditions de traitement de l'information sur les marchés. Cet article présente une recherche destinée à décrire le comportement informationnel des entreprises de taille moyenne en vue d'un développement international plus ou moins intense.

Une étude a été réalisée dont la méthodologie repose sur des méthodes descriptives mettant en évidence les caractéristiques de comportement informationnel d'entreprises exportatrices de taille moyenne.

Deux catégories de résultats, issus de deux phases méthodologiques, ressortent de cette étude :

- il existe une différence de comportement de gestion de l'information entre des entreprises peu exportatrices et des entreprises très exportatrices ;

- au-delà d'une appréciation dichotomique portant seulement sur le caractère plus ou moins exportateur que la moyenne du secteur étudié, il s'avère que certaines entreprises performantes développent une prise en compte rigoureuse de la ressource information alors que d'autres préfèrent exploiter les qualités relationnelles propres aux PME pour capter l'information et permettre l'anticipation.

La conclusion de l'article oriente le lecteur vers la validation de l'importance des systèmes d'information marketing dans les PME, mais aussi vers la prise en compte des spécificités de la PME pour dénouer le paradoxe informationnel évoqué en introduction.
Ce document est protégé par la loi sur le droit d'auteur. L'utilisation des services d’Érudit (y compris la reproduction) est assujettie à sa politique d'utilisation que vous pouvez consulter en ligne.

https://apropos.erudit.org/fr/usagers/politique-dutilisation/ 


\section{PME, gestion internationale et systèmes d'information marketing: au-delà des évidences technologiques}

Martine BOUTARY

École supérieure de commerce de Toulouse

\section{MOTS CLÉS}

\section{PME - Information - Développement international}

\section{RÉSUMÉ}

La connaissance des marchés a souvent été mise en évidence comme étant indispensable à la réussite internationale. Les structures exportatrices de taille moyenne sont particulièrement sensibles à ce problème : leur besoin informationnel est rendu important par des marchés géographiquement dispersés, alors que leurs systèmes d'information sont le plus souvent peu formalisés, peu sophistiqués et vite surchargés. La tentation d'un discours normatif est alors grande et de nombreux conseils de gestion de l'information, soutenus par les informaticiens, sont mis en place.

La problématique abordée est celle d'une performance sur le marché international soumise à des conditions de traitement de l'information sur les marchés. Cet article présente une recherche destinée à décrire le comportement informationnel des entreprises de taille moyenne en vue d'un développement international plus ou moins intense.

Une étude a été réalisée dont la méthodologie repose sur des méthodes descriptives mettant en évidence les caractéristiques de comportement informationnel d'entreprises exportatrices de taille moyenne.

\section{L'AUTEURE}

Martine Boutary est professeure à l'École supérieure de commerce de Toulouse et responsable du mastère spécialisé "Intelligence économique et stratégie d'entreprise ". Adresse: 20 , boulevard Lascrosses, B.P. 7010, 31068 Toulouse Cedex 7, France. Courriel : < m.boutary @esc-toulouse.fr > 
Deux catégories de résultats, issus de deux phases méthodologiques, ressortent de cette étude:

- il existe une différence de comportement de gestion de l'information entre des entreprises peu exportatrices et des entreprises très exportatrices;

- au-delà d'une appréciation dichotomique portant seulement sur le caractère plus ou moins exportateur que la moyenne du secteur étudié, il s'avère que certaines entreprises performantes développent une prise en compte rigoureuse de la ressource information alors que d'autres préfèrent exploiter les qualités relationnelles propres aux PME pour capter l'information et permettre l'anticipation.

La conclusion de l'article oriente le lecteur vers la validation de l'importance des systèmes d'information marketing dans les PME, mais aussi vers la prise en compte des spécificités de la PME pour dénouer le paradoxe informationnel évoqué en introduction.

\section{ABSTRACT}

International success is closely linked to market knowledge. Small and medium-sized export firms are particularly aware of this problem: their need for information is important due to geographically dispersed markets, when their information systems are very often little formalized, little sophisticated and quickly overloaded. Temptation for a normative speech is then high and a system of management and information system based on recommendations from computer analysis is often implemented.

Our problematic is an international performance based on conditions of market information analysis. This article presents a research work describing the informational behavior of medium-sized enterprises which aim at an international development more or less intense.

A survey has been achieved, with a methodology based on descriptive methods to highlight features of informational behavior of medium-sized exporters.

Two categories of results, stemming from two methodological phases, emerge from this survey:

- a difference of behavior for information management exists between the few exporter enterprises and the more important ones;

- beyond a dichotomous appraisal focusing only on the studied sector as exporting more or less than average, it turns out that certain effective enterprises rigourously develop their information resource whereas others prefer to exploit the relational qualities specific to SME's in order to retrieve information and allow anticipation.

The conclusion orients the reader toward the validation of the importance of information marketing systems in the SME's but also toward the importance of SME's specificities to unknot the informational paradox evoked in introduction. 


\section{RESUMEN}

A menudo el conocimiento de los mercados, se ha señalado como indispensable en el éxito internacional. Las sociedades exportadoras de tamaño medio son especialmente sensibles a esta problemática: su necesidad de información es especialmente importante para los mercados dispersados geográficamente, considerando que sus sistemas de información a menudo son informales, poco sofisticados y son rápidamente sobrecargados. La tentación de un discurso normativo es entonces grande y muchos consejos sobre gestión de la información, sostenidos por informáticos, son llevados a cabo.

El problema abordado es el del éxito de estas empresas en el mercado internacional dependiente del modo de tratar la información. El objetivo de la publicación propuesta es la presentación de una investigación realizada para describir la gestión de la información de las empresas medianas para su desarrollo internacional.

Dos categorías de resultados, producto de dos etapas metodológicas, son obtenidas de este estudio:

- existe una gran diferencia en la gestión de información entre las empresas poco exportadoras y las empresas muy exportadoras;

- más allá de una evaluación dicotómica, de acuerdo al mayor o menor grado de exportación en relación al promedio del sector estudiado, esta investigación demuestra que ciertas empresas eficaces desarrollan una gestión de información rigurosa, mientras que otras prefieren explotar las cualidades relacionales, propias a las empresas medianas, para obtener la información y permitir la anticipación.

La conclusión de este artículo orienta al lector hacia la validación de la importancia de la información para el éxito internacional, pero también muestra que la consideración de las características propias de estas empresas permite resolver la paradoja de la información evocada en la introducción.

\section{ZUSAMMENFASSUNG}

Die Marktkenntniss wird oft als unabdingbarer Faktor für den internationalen Erfolg einer Unternehmung betrachtet und steht somit im Zentrum der Ueberlegungen. Die Exportstrukturen von mittleren Unternehmen reagieren besonders sensibel auf dieses Problem: Bedingt durch die stark zerstreuten geographischen Märkte ist das Bedürfnis von mittleren Unternehmen nach Informationen stark gestiegen. Diese Problematik ist von umso grösserer Tragweite, weil die Informationssysteme wenig formalisiert, nur ungenügend entwickelt und schnell ausgelastet sind. Der Versuch Richtlinien und Vorschläge anzubringen für das Informationsmanagement, meistens unterstützt von Informatikern, ist vermehrt zu betrachten.

Im Vordergrund steht die Exportleistung auf dem internationen Markt und die anforderungsreichen Bedingungen der Marktinformationen. Der vorgeschlagene Artikel bezweckt, eine Untersuchung darzustellen, welche das Informationsverhalten von mittleren Unternehmen beschreibt. Dabei handelt es sich um Betriebe mit unterschiedlicher Intensität in ihrer internationalen Entwicklung. 
Die Studie über die Merkmale des Informationsverhalten von exportorientierten mittleren Unternehmen hatte beschreibenden Charakter und lieferte zwei Ergebnisse:

- Es existiert ein unterschiedliches Verhalten beim Informationsmanagement zwischen wenig exportorientierten und stark exportorientierten Untermehmen.

- Bei den mehr oder weniger exportierenden Unternehmen konnte festgestellt werden, dass einige Betriebe dem Informationsmanagement sehr grosse Bedeutung beipflichten. Während andere Unternehmen die qualitativen Beziehungen bevorzugen, um zu den wichtigen Informationen zu gelangen.

Das Fazit dieses Artikels bestätigt dem Leser die Wichtigkeit von Marktinformationssystemen bei kleinen und mittleren Unternehmen (KMU), betont aber auch die Bedeutung der speziellen Gegebenheiten der KMU beim Informationsverhalten: Einerseits benötigen sie Marktinformationen, andererseits sind ihre Anstrengungen nicht ausreichend beim Aufbau von Informationssystemen.

\section{L'information internationale: la PME exportatrice en attente de modèle?}

Malgré l'importance économique du tissu des PME, on ne peut que rester préoccupé par la faiblesse des résultats de ces entreprises sur le marché international (Besh et Guieu, 1993 ; Ducheneaut, 1996; Julien et al., 1998). Si les PME représentent $95 \%$ du nombre des exportateurs, elles ne génèrent que $33 \%$ des exportations françaises, et ce, essentiellement vers l'Union européenne (Ducheneaut, 1996). En 1998, sur les 2,3 millions d'entreprises en activité, 115000 seulement ont développé ou poursuivi un courant d'affaires international (Bourgeois et Estival, 2000).

Il est donc légitime de la part des chercheurs d'approfondir les recherches sur l'origine des performances de ces structures. Ces travaux, au-delà de la difficile opérationnalisation du concept de performance export (Fenwick et Amine, 1979; Chryssochoidis, 1992), permettent d'identifier les facteurs déterminants du succès ou de l'échec de ces entreprises sur le marché international (Aaby et Slater, 1988; Denis, 1990 ; Gemunden, 1993). Ils soulignent la diversité des variables explicatives des performances internationales (Boyer, 1993), provenant de caractéristiques de structure ou de caractéristiques environnementales. Dans ce contexte, tous les éléments de la politique marketing de ces entreprises sont cités (Cavusgil et Nevin, 1981 ; Roux, 1991 ; Denis, 1990). L'un d'eux, souvent mentionné mais peu analysé, apparaît de plus en plus régulièrement (Algoe, 1992 ; Julien et al., 1998) : la gestion de l'information.

D'autres auteurs étudient la performance sous l'angle de la mondialisation et mettent aussi en évidence l'importance de la gestion de l'information (Madhok, 1996 ; Julien et al., 1998) comme source d'avantage concurrentiel de l'entreprise. 
La maîtrise de l'information et de la connaissance est alors, dans le cadre de la théorie des ressources, un facteur explicatif de la réussite des organisations dans la mesure où, dans un contexte d'analyse stratégique, elle permet à l'entreprise de mieux repérer son environnement et d'établir des stratégies adaptées. La survie des PME exportatrices, majoritairement orientées vers des stratégies de niche leur permettant d'exploiter de façon optimale un savoir-faire souvent unique et un fort degré de spécialisation (Saporta, 1986), passe notamment par la capacité de modifier très rapidement une première orientation ou de suivre, voire d'anticiper, les exigences mouvantes de certains marchés (Kashani, 1995). Cela requiert des informations (Gemunden, 1991), mais aussi beaucoup de souplesse (Lesca, 1992) pour l'intégration de celle-ci dans le processus de recherche, de production et de commercialisation de l'entreprise.

Directement centrées sur «l'objet PME » ou plus généralement orientées vers l'analyse stratégique, l'ensemble de ces recherches relèvent une situation paradoxale, issue de la superposition du caractère réduit des structures de PME et du caractère géographiquement très large de leur environnement. Celui-ci engendre un besoin informationnel plus important et plus diversifié que ceux des entreprises non exportatrices (Monnoyer, 1993), alors que le système d'information marketing de ces structures reste très informel (Herrmann, 1998), éloigné de la définition classique comme « réseau de relations structurées où interviennent des hommes, des machines, des procédures, qui a pour objet d'engendrer un flux ordonné d'informations pertinentes, provenant de sources internes et externes à l'entreprise et destiné à servir de base aux décisions Marketing (Evrard et Lemaire, 1993). Pourtant, l'identification et la localisation de niches de marché, la connaissance du comportement des acteurs étrangers, de la législation locale, des aspects financiers sont dans ce cadre autant d'informations indispensables à maîtriser pour des prises de décision pertinentes. Les informations classiques - de type comptable par exemple - essentiellement introverties, en provenance et en direction de l'entreprise, reflétant le passé et concernant des opérations précises, doivent céder le pas à des informations extraverties, très liées à l'environnement (Bouquin, 1989). Celles-ci, fournies par des sources presque toujours extérieures à l'entreprise, ont le plus souvent une nature prospective et globale et se présentent sous une forme plus qualitative que quantitative. Elles sont par ailleurs marquées par des codifications variées, linguistiques ou culturelles (Usunier, 1992) et soumises à des difficultés d'interprétation (Mayere, 1995). Dans un univers ainsi élargi, la connaissance intuitive souvent utilisée par les dirigeants de PME est moins efficace et devra être complétée par des données extérieures. Il faut alors savoir capter l'information, par l'intermédiaire de ce que certains auteurs ont appelé des «intrusions » dans l'environnement (Baumard, 1996) et par la vigilance (Amabile, 1994) permanente à des signaux divers. 
Pareille exigence suppose d'importantes capacités de collecte et de traitement des informations pouvant être soutenues dans le plus grand nombre d'entreprises, par le développement des potentialités informatiques et des technologies de l'information (Raymond, 1990). Or, malgré l'existence des outils nécessaires, malgré l'importance reconnue de l'information pour le développement international et malgré l'abondance d'informations disponibles (Nonaka, 1994), les dirigeants de PME éprouvent encore de réelles difficultés à résoudre le problème du traitement de cette information (Raymond, 1984). Dans un environnement sur lequel ils n'ont que peu de contrôle et qu'ils doivent connaître, ces dirigeants développent des systèmes d'information très informels, vite saturés par la surcharge de données (Raymond, 1984 ; Julien, 1994) et structurés autour d'une forte tradition orale et d'une forte valorisation des connaissances tacites (Nonaka, 1990; Baumard, 1996): le sentiment de maîtriser l'information par la connaissance personnalisée des différents maillons de la chaîne économique sécurise autant les dirigeants qu'un haut degré de formalisation de l'information. Dans ces systèmes, les données formalisées ne suffisent pas : élaborées pour répondre à des besoins exprimés par d'autres, elles ne peuvent être appropriées par les PME.

La tentation est alors grande de conclure à une insuffisance : insuffisance de moyens, insuffisance d'organisation et de temps pour capter et utiliser l'information indispensable à la survie et au développement de l'activité internationale de ces entreprises. La solution passe alors par l'élimination de la surcharge par le tri et la compression pour compenser l'insuffisance de moyens de traitement ou inversement par une multiplication des données chargées de combler l'insuffisance de contenu. Les organismes voués à l'appui aux entreprises ont, par exemple, multiplié les données, d'une part (les sources d'information sur les marchés internationaux sont nombreuses et de mieux en mieux répertoriées), et les points d'accès à ces données, d'autre part (un exemple récent est, en France, l'ouverture de Centres régionaux d'information internationale).

Mais les difficultés sont toujours présentes (Boutary, 1998). La multiplication des données est telle que leur organisation devient un sujet de préoccupation. Les contraintes humaines (effectifs réduits) ou financières empêchant une mise à plat des besoins et de plans de recherche précis, deux comportements apparaissent (Boutary, 1998) : l'abandon de toute procédure de sélection - amenant rapidement l'entreprise à l'asphyxie informationnelle - ou, inversement, un cadrage étroit de tout le système d'information qui devient alors une sorte de simple armoire de stockage pour informations très vite obsolètes. Rien ne semble guider les dirigeants, la méconnaissance des processus de mesure de la rentabilité des investissements immatériels tels que l'information confortant ces comportements (Baile et Sole, 1995).

La réflexion se tourne vers la mise en place de systèmes d'information à partir d'une approche centrée sur les outils et les ressources disponibles. Il est à cet endroit 
important de revenir sur la définition des systèmes d'information. Ce terme, apparu dans les années 1950 (Monnoyer, 1995), est défini en 1974 par Davis (Davis, 1974) comme « un système intégré homme-machine qui fournit les informations supportant les opérations, la gestion et les prises de décision dans une organisation sociale ». L'originalité de cette définition tenait alors à sa volonté d'isoler les fondements conceptuels des systèmes d'information des technologies mises en œuvre pour la constitution ou le fonctionnement de ces mêmes systèmes. Informatique et information restent très liées, sans que la machine n'apparaisse comme la condition suffisante d'apparition de l'information. Grâce à cette approche, on dispose d'une construction théorique permettant de rendre compte du couplage d'un système de pilotage sur un système opérationnel. Comme le souligne Le Moigne (1986), « la base conceptuelle des systèmes d'information est donc le modèle cybernétique de l'organisation, articulée autour d'un système piloté et d'un système pilotant ». Cette approche s'appuie sur l'hypothèse de l'existence d'une entreprise pyramidale, hiérarchisée, dans laquelle les flux d'information sont identifiés, codifiables et canalisés, maîtrisés par certains acteurs et méconnus de certains autres.

Or, les PME ne sont pas des structures hiérarchisées et respectant le modèle cybernétique, notamment dans le cadre de la prise de décision (Chapellier, 1994). Les flux informationnels ne sont pas exactement des flux identifiés et codifiables. Les contraintes financières et humaines déjà évoquées amènent ces organisations, très soucieuses de réactivité, à délaisser le seul recours à des données formalisées (études, par exemple) et donc facilement codifiables, pour une utilisation plus complexe d'informations informelles et spéculatives les aidant à mieux comprendre de façon implicite la nature de leur environnement et de son évolution. L'appréciation de leurs systèmes d'information comme systèmes incomplets, en construction et freinés par un ensemble de contraintes, semble alors très soumise à un schéma d'évaluation classique, à partir de deux présupposés (Ermes, 1994) : les problèmes sont bien structurés et peuvent s'exprimer complètement de façon objective et stable dans le temps. L'approche est normative, supposant que l'entreprise, pour être performante, doit mettre en place des procédures et développer des systèmes d'information sophistiqués. Il s'agit notamment, à partir d'une connaissance informatique de plus en plus riche et capable d'intégrer de plus en plus de paramètres, de constituer les supports nécessaires à la prise de décision des managers (Blili et Raymond, 1994).

De récentes recherches invalident la proposition selon laquelle les entreprises performantes posséderaient les systèmes d'information les plus sophistiqués (Julien et al., 1998). Nous souhaitons revenir sur ce modèle d'un système enflé, sophistiqué par les apports informatiques et télécommunicationnels, devenu procédural. Nos questions sont les suivantes : faut-il vraiment multiplier les contacts pour être plus performant ? Faut-il vraiment tout mettre sous procédure, de l'analyse des besoins informationnels à leur traitement? 
La problématique de notre recherche est celle d'une performance à l'international soumise à des conditions de gestion de l'information, gestion elle-même soumise aux spécificités des acteurs. Nous focaliserons notre attention sur les pratiques effectives des PME en matière de gestion de l'information, avec l'objectif de décrire ce qui se passe dans ces entreprises et d'éviter les conclusions comparatives, notamment avec des grandes entreprises.

Deux hypothèses sont proposées, l'une portant sur la liaison de la performance des PME exportatrices et de leur capacité et de leur intérêt à capter l'information externe à la structure, l'autre portant sur la liaison de la performance de ces entreprises et leur capacité à intégrer des informations nombreuses, certaines formalisées et d'autres non, en fonction de besoins plus ou moins prédéterminés.

Les deux hypothèses de recherche seront ainsi formulées :

$\mathrm{H} 1$ : les entreprises de taille moyenne exportatrices les plus performantes sur leurs marchés internationaux recherchent plus l'information que les entreprises de mêmes caractéristiques mais moins performantes sur les marchés internationaux;

$\mathrm{H} 2$ : les entreprises de taille moyenne exportatrices et performantes utilisent plus que les moins performantes des systèmes d'information ouverts sur les marchés et capables de capter des informations non programmées.

Nous montrerons que certaines caractéristiques de gestion de l'information sont liées à la performance des PME exportatrices, mais que cette hypothèse est validée de façon nuancée : ce n'est pas la seule sophistication du système d'information qui garantit une meilleure performance, car interviennent les spécificités organisationnelles des entreprises de taille moyenne.

\section{Méthodologie de la recherche}

\subsection{Constitution de l'échantillon}

Les recherches sur les PME ont souligné l'hétérogénéité de ce concept et mis en évidence la considération de l'ensemble de la population comme l'une des limites à l'exploitation des résultats obtenus (Ammeux, 1984 ; Julien, 1993 ; Le Vigoureux, 1995).

Pour limiter ce biais, l'approche empirique destinée à qualifier le comportement informationnel d'entreprises de taille moyenne a été limitée aux entreprises françaises exportatrices du secteur industriel, ayant un effectif de 100 à 200 personnes. 
Au-delà du choix d'entreprises françaises effectué pour de simples raisons pratiques, les choix méthodologiques correspondent aux raisons suivantes :

- l'appartenance à un secteur d'activité n'est pas sans influence sur le comportement des entreprises au regard de leur développement international (Roncin, 1982). Cette considération conduit à aborder avec prudence les statistiques globales et rend nécessaire une observation sectorisée. Le secteur industriel a été choisi parce qu'il est en France le plus exportateur, tant par le nombre d'entreprises concernées que par le taux moyen du chiffre d'affaires export par rapport au chiffre d'affaires global (Ducheneaut, 1996);

- la variable «taille de l'entreprise » a suscité, dans l'univers de la gestion, de nombreuses interrogations et par là même, de nombreuses recherches qui ont souligné l'importance de cette variable sur le degré d'internationalisation des entreprises et sur le mode de gestion de l'information (Avisco Conseil, 1988; Ducheneaut, 1996). De précédentes recherches montrent que les entreprises ayant un effectif inférieur à 100 personnes ou supérieur à 200 ont un ratio chiffre d'affaires export / chiffre d'affaires global plus important que les entreprises de taille moyenne. De la même façon, la valeur ajoutée par tête d'effectif se situe sur une courbe en $U$ pour les entreprises exportatrices: la valeur minimale se situe dans la classe des 100 à 199 salariés (Roncin, 1982).

Il paraît donc pertinent, dans le cadre d'une recherche sur la performance, de porter attention à cette classe d'entreprises, qui ont perdu la spontanéité des plus petites et n'ont pas acquis les procédures des plus grandes pour développer leur action internationale.

Enfin, pour répondre à l'objectif de la recherche, l'étude doit porter sur deux populations à la fois semblables et différentes: semblables, car exportatrices et appartenant au secteur industriel, différentes par les résultats obtenus en matière de développement international.

Nous avons éliminé les entreprises effectuant seulement des opérations ponctuelles, grâce au hasard et à des contacts étrangers qu'elles peuvent avoir dans le cadre d'une activité commerciale strictement nationale. C'est le cas d'entreprises exposant dans des salons internationaux en France, exploitant les retombées du salon sans jamais se déplacer ou adapter leur politique commerciale aux spécificités de la clientèle étrangère. La recherche portant sur la démarche informationnelle des entreprises exportatrices, il semblait pertinent d'éliminer les entreprises qui ne font «que » gérer les occasions d'affaires à partir de peu d'informations (Joyal, 1993).

La sélection a donc porté sur les entreprises exportant depuis au moins trois ans. Il a été demandé à l'ensemble des répondants de préciser leur chiffre d'affaires 
export sur la période des trois dernières années. L'absence de chiffre au cours de l'une des trois années de référence entraînait le rejet du questionnaire recueilli.

Deux sous-populations sont identifiées, l'une plus exportatrice que la moyenne du secteur, l'autre moins exportatrice. Le pourcentage moyen du chiffre d'affaires exporté dans le secteur industriel est de $20,8 \%^{1}$, pour une plus grande facilité de calcul et de représentation, la valeur distinctive entre les deux sousgroupes est fixée à $20 \%$. Seront alors qualifiées de «moins exportatrices» les entreprises exportant moins de $20 \%$ de leur chiffre d'affaires global, et d'entreprises «plus exportatrices » celles qui exportent plus de $20 \%$ de leur chiffre d'affaires global' ${ }^{2}$.

La population correspondant à l'ensemble de ces critères est de 682 entreprises ${ }^{3}$.

Un questionnaire destiné à identifier l'entreprise, ses performances à l'export et ses pratiques informationnelles a été envoyé par voie postale avec rappel téléphonique à l'ensemble de cette population. Quatre-vingt-douze questionnaires ont pu être traités ; ils se répartissent entre 42 entreprises exportant moins de $20 \%$ de leur chiffre d'affaires et 50 entreprises exportant plus de $20 \%$ de la totalité de leur chiffre d'affaires.

\subsection{Choix des indicateurs}

L'enquête présentée dans cet article a été précédée d'une étude exploratoire portant sur le concept d'information au sein des entreprises de taille moyenne (Boutary, 1998).

Les indicateurs choisis pour rendre opérationnelles les variables « recherche d'informations extérieures » et « ouverture des systèmes d'information » sont issus de la littérature et de l'étude exploratoire préalablement effectuée.

1. Ce taux est le pourcentage du chiffre d'affaires export par rapport au chiffre d'affaires global donné par le SESSI à partir d'un échantillon d'entreprises françaises du secteur industriel ayant un effectif de 20 à 500 salariés (Ducheneaut, op. cit., p. 465).

2. Les entreprises plus exportatrices seront par la suite notées E+, les entreprises moins exportatrices seront notées $\mathrm{E}-$, notamment pour la mise en évidence des résultats de l'étude.

3. Données Kompass France 1995 : 5446 entreprises ont un effectif compris entre 100 et 199 salariés ; 25666 entreprises sont exportatrices, toutes tailles et tous secteurs confondus ; le croisement de ces deux critères donne une population de 2309 entreprises. En ne prenant que les entreprises industrielles sélectionnées à partir des codes NAF 20 à 24 et 29 à 35 , on obtient 682 entreprises. 


\subsubsection{Recherche d'information}

L'abondance de l'information propre au développement international a été soulignée dans la littérature. Celle-ci mentionne plusieurs indicateurs destinés à mesurer la recherche d'information (de Mattei Pasture, 1993) : l'utilisation des services publics, de sources externes, des agents de l'entreprise, des fédérations professionnelles, des salons et des médias.

Ont été retenus, après validation par l'étude exploratoire, les indicateurs suivants pour la mesure de la recherche d'information:

- les abonnements à des revues françaises ou étrangères (Reid, 1984 ; Hunt et Zartarian, 1990 ; Roux, 1991 ; Lesca, 1992) comme mesure de l'intérêt que l'entreprise porte à l'information en provenance de l'extérieur de sa propre structure pour une meilleure connaissance des marchés;

- les relations des entreprises exportatrices avec les organismes centrés sur le développement international (Cavusgil, 1984 ; Monnoyer, 1993). Ces relations ont été mesurées par l'existence, dans l'année précédant le remplissage du questionnaire, d'un contact effectif pour une question concernant le développement international entre l'entreprise concernée et les organismes suivants : la Compagnie française d'assurance pour le commerce extérieur (COFACE), le Centre français du commerce extérieur (CFCE), les chambres de commerce et d'industrie, un organisme de normalisation international (NOREX), l'Agence nationale pour la valorisation de la recherche (ANVAR), les banques et les fédérations professionnelles.

Nous souhaitions, par ailleurs, vérifier si cette recherche était un acte véritablement engagé de la part de l'entreprise. Deux autres indicateurs ont été utlilisés, l'un déjà présent dans la littérature (Cooper et Kleinschmidt, 1985 ; Blili et Raymond, 1994 ; Kashani, 1995), l'existence d'un budget information, l'autre issu de l'étude exploratoire, la prévision par le dirigeant d'une évolution prochaine de ce budget.

\subsubsection{Ouverture des systèmes d'information}

La mesure de l'ouverture des systèmes d'informations - correspondant à une sélection faible des informations reçues - sera effectuée grâce à des indicateurs issus de la littérature : la perception de l'utilité de l'information (Cavusgil et Godiwalla, 1982; Gale, 1992), la nature des informations payées et des informations recherchées (Roux, 1991 ; Algoe, 1992), mais principalement des entretiens exploratoires : la perception du système formalisé comme contrainte, la perception d'une nécessaire formalisation pour éviter la «noyade informationnelle», les raisons justifiant le paiement d'informations (Boutary, 1998). 
Les variables « recherche d'information » et « ouverture des systèmes d'information » sont ainsi rendues opérationnelles. C'est la convergence du faisceau d'indicateurs choisis vers chacun des concepts étudiés qui permettra de valider l'hypothèse.

Le traitement des données nous a amenée à recourir à une première approche bivariée pour vérifier les liaisons entre l'importance de l'activité internationale des entreprises et certaines modalités de gestion de l'information. Le test du khi carré a été systématiquement utilisé pour vérifier l'indépendance statistique des variables de chaque tableau de contingence. Dans un deuxième temps, dans un souci d'identification de comportements informationnels, une typologie a été effectuée. Celle-ci a été précédée d'une analyse factorielle des correspondances multiples sous Statistical Package for Social Sciences (SPSS) destinée à dégager les dimensions principales des comportements étudiés.

\section{Résultats}

\subsection{Recherche de l'information internationale: des comportements différents selon le niveau de performance export}

\subsubsection{Un intérêt général pour les revues françaises... moins soutenu pour la presse internationale}

Parmi les entreprises interrogées, 97,8\% sont abonnées à des revues françaises. Ce résultat, indépendant du taux d'exportation, confirme un intérêt global de l'ensemble des entreprises à l'information externe sur leur environnement. En revanche, seulement $57,6 \%$ des entreprises sont abonnées à des revues étrangères.

TABLEAU 1

Abonnement des entreprises à des revues étrangères

\begin{tabular}{|c|c|c|}
\hline & $\begin{array}{l}\text { Entreprises abonnées } \\
\text { à des revues étrangères }\end{array}$ & $\begin{array}{l}\text { Entreprises non abonnées } \\
\text { à des revues étrangères }\end{array}$ \\
\hline E- & $21,40 \%$ & $78,60 \%$ \\
\hline E+ & $88 \%$ & $12 \%$ \\
\hline$\chi^{2}$ & \multicolumn{2}{|c|}{0,00000} \\
\hline
\end{tabular}

Ce résultat est lié à la performance export : $21,4 \%$ des entreprises moins exportatrices sont abonnées à des revues étrangères contre $88 \%$ dans le cas des entreprises plus exportatrices.

L'information étrangère est plus difficile d'accès - notamment à cause de la contrainte linguistique. Les entreprises moins exportatrices ne voient pas leur intérêt 
et, plus généralement, ne connaissent pas les possibilités d'information en ce domaine. En revanche, les entreprises plus exportatrices utilisent massivement ces sources d'information pour mieux connaître leurs marchés - plusieurs d'entre elles ont précisé une préférence pour la presse étrangère pour des raisons de fiabilité des sources.

\subsubsection{Des contacts inégaux avec l'environnement}

Les entreprises ont été interrogées sur l'identité des organismes avec lesquels elles ont communiqué au cours de la dernière année de référence au sujet de leur développement export. Deux groupes semblent se « détacher» :

- d'une part, la Compagnie française d'assurance pour le commerce extérieur (COFACE) et les banques, que les entreprises ont citées dans respectivement $65,2 \%$ et $62 \%$ des cas ;

- d'autre part, le Centre français du commerce extérieur (CFCE) cité par $37 \%$ des entreprises, les postes d'expansion économique cités par $47,8 \%$ des entreprises, les chambres de commerce citées par $35,9 \%$ des entreprises, les fédérations professionnelles citées par $42,4 \%$ des entreprises, l'Agence nationale pour la valorisation de la recherche (ANVAR) citée par 28,3\% des entreprises et la NOREX (Normes à l'exportation) citée par $4,3 \%$ des entreprises.

Les pourcentages relevés montrent que les deux groupes correspondent à des taux de sollicitation différents. Mais une analyse plus précise permet de constater que les résultats concernant le premier groupe (COFACE - banques) sont liés au taux d'exportation des entreprises : la COFACE a été en relation avec $50 \%$ des entreprises moins exportatrices et $78 \%$ des entreprises plus exportatrices ; le réseau bancaire a été en relation avec $40,5 \%$ des entreprises moins exportatrices et $80 \%$ des entreprises plus exportatrices.

En revanche, les résultats concernant le Centre français du commerce extérieur, les postes d'expansion économique, les chambres de commerce, les fédérations professionnelles, l'Agence nationale pour la valorisation de la recherche et la NOREX ne sont pas liés à l'importance de l'activité export des entreprises.

Ces résultats peuvent surprendre : le CFCE est un centre d'information uniquement tourné vers l'appui des entreprises en matière de développement export, tout comme le sont les postes d'expansion économique à l'étranger. Comment expliquer alors que ces organismes-là ne soient pas systématiquement en relation avec les entreprises moyennes exportatrices ? Le sens de la recherche oriente cette interrogation vers une autre formulation: quels sont les éléments de la démarche informationnelle des entreprises exportatrices de taille moyenne qui les poussent plutôt vers la COFACE et le réseau bancaire que vers le CFCE et les postes d'expansion? 
Une première explication pourrait être que la COFACE et le réseau bancaire fournissent des informations de type « information de fonctionnement " (modes de paiement, assurance, mise sur le marché d'un produit déterminé, etc.) nécessaires aux entreprises plus exportatrices. Cela pourrait expliquer la liaison de l'activité export de l'entreprise, mais ne peut expliquer pourquoi les organismes voués à l'appui informationnel pour le développement international ne sont pas plus massivement consultés par les entreprises dans leur ensemble.

L'expression du besoin informationnel est par nature complexe. Avoir une bonne information a longtemps été assimilé à « avoir beaucoup d'informations » et il est effectivement impressionnant de constater le volume de l'information disponible dans les structures publiques. Malheureusement, celle-ci ne semble pas en phase avec les besoins - souvent peu ou mal exprimés - des entreprises exportatrices. Face à des situations peu prévisibles, pour lesquelles elles n'ont pas a priori la capacité de déterminer la totalité de leurs besoins en matière d'information, les entreprises vont rechercher et privilégier des partenaires capables de leur fournir, dans un climat de confiance élevé, des informations sélectionnées et déjà partiellement analysées ; il ne s'agit plus d'agréger les données, mais de les filtrer, de les commenter en fonction des besoins de l'entreprise, dans un but d'appropriation d'une nouvelle connaissance par le dirigeant. Le concept de fiabilité et de richesse des sources sera alors très valorisé, ce que semblent avoir compris le réseau bancaire et la COFACE.

Trois points peuvent être mis en évidence :

- la nature des contacts est liée à l'importance de l'activité internationale des entreprises ;

- les contacts sont moins importants avec les organismes essentiellement pourvoyeurs d'informations générales non personnalisées (infos - marchés, etc.);

- les contacts sont plus importants avec les organismes revendiquant une démarche commerciale orientée vers le développement du service et la personnalisation de la relation.

\subsubsection{Une conscience floue de l'existence financière d'un poste "Information» et de son évolution}

Seulement $28,3 \%$ des entreprises ont déclaré avoir un budget information, alors que $63 \%$ déclarent avoir déjà payé de l'information! Ces résultats sont liés à l'importance de l'activité internationale de l'entreprise: seulement $11,9 \%$ des entreprises moins exportatrices ont un budget information (mais elles sont $40,5 \%$ à payer de l'information), alors que $42 \%$ des entreprises plus exportatrices ont un budget information (mais elles sont $82 \%$ à payer de l'information). Ce résultat met en évidence une plus grande consommation d'information par les entreprises plus 
TABLEAU 2

Existence et projet de développement d'un budget information

\begin{tabular}{|c|c|c|c|c|}
\hline & $\begin{array}{l}\text { Existence d'un } \\
\text { budget }\end{array}$ & $\begin{array}{l}\text { Inexistence d'un } \\
\text { budget }\end{array}$ & $\begin{array}{l}\text { Développement } \\
\text { prévu d'un } \\
\text { budget info }\end{array}$ & $\begin{array}{c}\text { Pas de } \\
\text { développement } \\
\text { prévu }\end{array}$ \\
\hline E- & $11,9 \%$ & $88,1 \%$ & $19 \%$ & $81 \%$ \\
\hline E+ & $42 \%$ & $58 \%$ & $42 \%$ & $58 \%$ \\
\hline$\chi^{2}$ & \multicolumn{2}{|c|}{0,00141} & \multicolumn{2}{|c|}{0,01826} \\
\hline
\end{tabular}

exportatrices - ce qui va dans le même sens que les apports de la littérature quant au développement des flux informationnels en fonction de l'exposition internationale - mais souligne aussi la difficulté d'attribution a priori d'un budget «information » peut-être due à une prise de conscience encore insuffisante de la nature et de la valeur de ce poste.

Deux remarques nous paraissent pertinentes dans ce cadre :

- l'attribution et la gestion d'un budget information dépendent de l'importance de l'activité internationale de l'entreprise;

- les entreprises plus exportatrices, soumises à une pression plus importante quant à la nécessité de gérer de l'information, sont plus nombreuses que les moins exportatrices à payer de l'information et à prévoir un budget sur ce poste.

L'idée même du budget information est donc tout à fait confuse. Ce que traduisent les chiffres est bien la nature complexe de l'information: quelle est la part d'un budget « salons » qui va passer dans le budget « information » ? La participation à des missions est-elle à affecter à la prospection dans son intégralité ou peut-on en affecter une partie à un poste « information »? Si définir l'information est une tâche complexe, il en va de même pour la définition d'un poste budgétaire spécifiquement dédié.

Au-delà de l'existence actuelle d'un budget «information », l'interrogation porte sur le choix du développement de ce budget par les dirigeants interrogés. Une telle orientation peut en effet indiquer une sensibilité récente à la notion d'information ou une prise de conscience de l'utilité de l'information dans un environnement fortement concurrentiel.

Dans les deux cas de figure, cela semble positif pour la mise en place dans l'avenir d'une politique de gestion de l'information. En ce qui concerne le développement de ce budget, $31,5 \%$ des dirigeants interrogés répondent positivement. Ce pourcentage est lié au taux d'exportation des entreprises : $19 \%$ des entreprises moins exportatrices souhaitent développer leur budget information alors que $42 \%$ des entreprises plus exportatrices ont cette même intention. 


\subsubsection{Recherche d'information, contacts et formalisation d'un budget: des comportements différenciés pour des performances équivalentes}

L'analyse typologique prolonge et approfondit notre recherche. Dans ce cadre, trois classes sont identifiées et comprennent respectivement :

- $33,7 \%$ de l'effectif pour la classe 1 ;

- $30,4 \%$ de l'effectif pour la classe 2 ;

- $35,9 \%$ de l'effectif pour la classe 3 .

L'analyse des trois classes repérées amène les remarques suivantes:

1. Les entreprises appartenant à la classe 2 sont caractérisées par:

- un taux d'abonnement à des revues étrangères relativement faible ( $21,2 \%$ contre $57,6 \%$ en moyenne);

- une fréquentation très inférieure à la moyenne générale des différents organismes de l'environnement international ;

- l'inexistence de tout budget information (aucune entreprise de cette classe n'a identifié un budget information) et la faible volonté d'investir sur ce thème-là $(96,4 \%$ des entreprises de cette classe n'envisagent aucun développement de ce poste);

- l'ensemble des entreprises de la classe 2 sont plus faiblement exportatrices que la moyenne : $14,3 \%$ d'entre elles exportent plus de $20 \%$ de leur chiffre d'affaires.

2. Les entreprises des classes 1 et 3 - représentant respectivement $33,7 \%$ et $35,9 \%$ de l'effectif étudié - sont en revanche plus exportatrices que la moyenne : $64,5 \%$ des entreprises de la classe 1 et $78,8 \%$ des entreprises de la classe 3 exportent plus de $20 \%$ de leur chiffre d'affaires.

Ces deux classes se ressemblent : les entreprises de la classe 1 et celles de la classe 3 sont très abonnées à des revues étrangères (respectivement 67,7 \% et 78,8\% des entreprises de ces deux classes). Elles ont payé des informations spécifiques pour leur développement international (respectivement $80,6 \%$ et $81,8 \%$ des entreprises de ces deux classes).

En revanche, les comportements sont divergents en matière de relation à l'environnement :

- les entreprises de la classe 1 cultivent les relations avec leur environnement de façon très importante. Dans le cadre précis de leur développement 
international, $83,9 \%$ ont eu des relations récentes avec les postes d'expansion, 93,5\% avec les banques, 96,8\% avec la COFACE, 83,9\% avec le CFCE, $64,5 \%$ avec les CCI. Seulement $25,8 \%$ d'entre elles ont un budget information et $32,3 \%$ souhaitent le développer prochainement ;

- les entreprises de la classe 3 agissent très différemment. Elles ont beaucoup moins de relations avec l'environnement : $36,4 \%$ ont eu des relations récentes avec les postes d'expansion, $54,5 \%$ avec les banques, $60,6 \%$ avec la COFACE, $12,1 \%$ avec le CFCE et $12,1 \%$ avec les CCI. En revanche, elles prévoient dans $54,5 \%$ des cas un budget information.

Deux types de comportement coexistent : d'une part, des entreprises très conviviales qui, pour obtenir les informations nécessaires à leur développement international, comptent plus sur la relation avec l'environnement que sur la formalisation d'un budget propre à l'information. Ce comportement a certainement quelque chose d'aléatoire, car la richesse des informations obtenues dépend des émetteurs et de la capacité de réception des entreprises à un moment donné. Ce comportement est proche d'un comportement d'entreprise de taille moyenne (forte tradition d'oralité, multiplication des contacts) déjà sensibilisée à la nécessité d'obtention du plus grand nombre d'informations possible.

Les entreprises de la classe 3 sont moins dépendantes de leur environnement, plus autonomes et rigoureuses dans leur système de surveillance et de recherche d'informations, plus engagées individuellement dans une stratégie de gestion de l'information et légèrement plus exportatrices que le groupe de la classe 1 .

Au-delà d'un constat permettant l'identification de comportements différents entre des entreprises plus exportatrices ou moins exportatrices que la moyenne, nous avons mis en évidence des différences à l'intérieur même du premier sous-groupe. Les entreprises plus exportatrices sont plus sensibles à l'information : elles multiplient les relations avec leur environnement, acceptent certains investissements sur ce poste et sont vigilantes aux évolutions de ce secteur. Leurs comportements ne sont cependant pas uniformes : si certaines privilégient les relations et les contacts avec l'environnement - notamment l'environnement institutionnel - laissant par là même, place à une forme d'aléa, d'autres préfèrent gérer avec plus de rigueur la ressource information.

Remarque est faite de deux comportements amenant à la réussite internationale : d'une part, l'exploitation des qualités relationnelles de la PME, d'autre part, la prise en compte rigoureuse de la ressource information. En revanche, les comportements peu sensibles à toute démarche informationnelle - que l'on trouve dans presque un tiers des entreprises - existent majoritairement dans des structures dont les résultats sur le marché international sont inférieurs à la moyenne. 


\subsection{Performance internationale et ouverture des systèmes d'information}

La deuxième hypothèse porte sur la capacité du système d'information marketing d'intégrer tout type d'information et sur l'existence d'une relation entre la performance export et l'ouverture du système d'information marketing : « Les entreprises de taille moyenne exportatrices et performantes utilisent des systèmes d'information ouverts sur les marchés, et capables de capter des informations non programmées. »

\subsubsection{Une intégration différenciée des informations non prévues ou non demandées}

«Il est nécessaire d'avoir un système de classement des informations extérieures à l'entreprise très précis et préformaté, sinon on se noie sous la diversité des informations »; 65,2\% des entreprises répondent « oui » à cette affirmation.

Ce comportement est lié à la performance export des entreprises : $83,3 \%$ des entreprises moins exportatrices estiment qu'il faut un préformatage du système de classement d'information, contre seulement $50 \%$ des entreprises plus exportatrices. Ces dernières acceptent plus facilement les informations imprévues, comprenant la nécessité de capter des informations qui, a priori, ne rentrent pas directement dans un champ de recherche trop strictement déterminé. Les entreprises moins exportatrices ont un comportement plus fermé et cherchent à maîtriser le classement de leurs informations. Elles laissent alors peu d'ouverture à l'imprévu ou à des signaux peu repérables dans le cadre d'une activité définie.

Par ailleurs, 26,1\% des entreprises sont d'accord avec l'affirmation suivante : «Une information utile est une information demandée. » Ce résultat est très lié à la performance export : $47,6 \%$ des entreprises moins exportatrices approuvent cette affirmation alors que seulement $8 \%$ des entreprises plus exportatrices pensent que l'information utile est une information demandée.

TABLEAU 3

Utilité de l'information

\begin{tabular}{|c|c|c|c|}
\hline & $\begin{array}{c}\text { Information utile = } \\
\text { information demandée }\end{array}$ & & $\begin{array}{l}\text { Information utile } \\
\text { information demandée }\end{array}$ \\
\hline$\overline{\mathrm{E}-}$ & $47,60 \%$ & & $52,40 \%$ \\
\hline $\bar{E}+$ & $8 \%$ & & $92 \%$ \\
\hline$x^{2}$ & & 0,00004 & \\
\hline
\end{tabular}

Ce résultat conforte les données de l'indicateur précédent. Les entreprises moins exportatrices travaillent avec plus d'aisance à l'aide de données dont elles maîtrisent l'origine - non en termes de sources mais en termes d'énoncés de besoins. 
L'information ne sera collectée ou achetée qu'en vue de répondre à un besoin : il y aura réponse s'il y avait demande, l'utilité restant perçue dans ce seul cadre.

\subsection{2. Études de marché et listes d'adresses: la majorité des investissements}

TABleaU 4

Types d'informations achetées

\begin{tabular}{lcc}
\hline Liaison & E- & E+ \\
\hline Achat Études de marché * & $21,40 \%$ & $44 \%$ \\
Achat Liste d'adresses * & $11,90 \%$ & $44 \%$ \\
Achat Normes & $16,70 \%$ & $30 \%$ \\
Achat Lettres confidentielles & $0 \% \%$ & $2 \%$ \\
Achat Recherche partenariale & $14,30 \%$ & $24 \%$ \\
\hline
\end{tabular}

* Marque la liaison existant avec l'indicateur de performance.

Les informations les plus achetées sont par ordre décroissant, les études de marché, les listes d'adresses, les normes, les recherches de partenariat (listes de fournisseurs, listes d'entreprises travaillant en amont ou en aval de la même filière) et, presque anecdotiquement, les lettres confidentielles.

Alors que les informations de type «études, normes » ou listes d'adresses (qui sont le plus souvent les listes d'acheteurs fournies par les postes d'expansion économique) devraient être prioritairement vendues aux entreprises moins exportatrices pour mettre en place ou conforter leur prospection, l'analyse montre au contraire que les entreprises plus exportatrices sont deux fois plus consommatrices que les autres de ce type d'informations.

Pouvons-nous en conclure que les entreprises les plus exportatrices sont en permanence à la recherche d'informations nouvelles, et ce, d'une façon très active ?

\subsubsection{La confidentialité: une justification de paiement de l'information réservée aux moins exportateurs}

Pour $57,6 \%$ des entreprises interrogées, payer une information se justifie par le caractère spécifique de l'information obtenue ; pour $46,7 \%$, c'est le professionnalisme de l'émetteur qui justifie le paiement; pour $31,5 \%$, c'est le caractère prospectif de l'information achetée et pour $27,2 \%$, c'est la confidentialité.

Un seul caractère est lié au taux d'exportation des entreprises : la confidentialité. Les entreprises moins exportatrices considèrent ce facteur comme justifiant le paiement de l'information - la «bonne information »-dans $40,5 \%$ des cas, alors que seulement $16 \%$ des entreprises très exportatrices valorisent ce caractère. 
TABLEAU 5

Justification d'achat d'information par la confidentialité

\begin{tabular}{|c|c|c|}
\hline & $\begin{array}{c}\text { La confidentialité } \\
\text { justifie l'achat. }\end{array}$ & $\begin{array}{l}\text { La confidentialité ne } \\
\text { justifie pas l'achat. }\end{array}$ \\
\hline E- & $40,5 \%$ & $59,5 \%$ \\
\hline E+ & $16 \%$ & $84 \%$ \\
\hline$x^{2}$ & \multicolumn{2}{|c|}{0,00857} \\
\hline
\end{tabular}

Cette plus grande exigence de confidentialité de la part des entreprises moins exportatrices paraît révélatrice d'une crainte à l'égard de la connaissance et de la circulation d'informations, crainte surmontée dans le cas des entreprises plus exportatrices utilisant des systèmes plus ouverts.

\subsubsection{L'expression du besoin informationnel pour le choix d'un marché}

Identifier un nouveau marché exige de la part d'une entreprise une démarche de recherche d'information ou la gestion d'occasions d'affaires. Dans le cas de notre échantillon, les entreprises interrogées sont toutes régulièrement exportatrices depuis au moins trois ans. Plusieurs comportements apparaissent cependant :

- certaines entreprises se limitent à répondre à des demandes ou agissent rapidement (pas d'étude ni de prospection), mais sans réflexion de long terme, elles n'ont alors pas réellement besoin d'un système d'information sophistiqué ;

- d'autres étudient les marchés, effectuent des repérages et agissent (étude puis prospection). Le premier temps de leur réflexion nécessite un apport riche de connaissances, entraînant alors la constitution de systèmes d'information marketing complexes et ouverts.

Le mode de fonctionnement des systèmes d'information est lié aux exigences attribuées à ces systèmes. Dans le cas d'un système considéré comme outil d'aide à la décision dans le sens le plus restrictif du terme, l'ouverture du système n'est pas capitale : le problème à résoudre - c'est-à-dire la décision à prendre - est identifié, les données à recueillir sont cadrées et existent dans un univers connu. Si le système d'information se rapproche d'un système de veille, sa capacité à capter des informations pour une prise de décision non programmée devra augmenter. L'univers est alors incertain, le repérage des problèmes et des décisions à prendre devient prioritaire.

Les réponses à la question «Comment choisissez-vous un marché étranger? » pourraient être présentées de la façon suivante. 
TABLEAU 6

Modalités de choix de nouveaux marchés

\begin{tabular}{lrc}
\hline & E- & E+ \\
\hline Étude puis prospection & $2,4 \%$ & $32 \%$ \\
Étude et prospection & $16,7 \%$ & $34 \%$ \\
Prospection puis étude & $11,9 \%$ & $12 \%$ \\
Pas d'étude : prospection & $64,3 \%$ & $20 \%$ \\
\hline
\end{tabular}

Ces résultats mettent en évidence la perception très différente du rôle de l'information dans les deux populations d'entreprises identifiées :

- le fait que $64,3 \%$ des entreprises moins exportatrices engagent une prospection sans étude préalable ni a posteriori est marquant: la force de décision d'un système traditionnel peu exportateur est encore très assise sur des schémas d'expérience adaptés à des environnements stables. Les dirigeants ne voient pas l'utilité des études, ils comptent prioritairement sur leur sentiment de connaissance de la situation, ce qui est acceptable dans le cas d'un environnement stable, mais plus difficile à gérer dans le cas d'un univers incertain. Les mutations de l'environnement sont alors délicates à prévoir.

Le pourcentage des entreprises plus exportatrices ne réalisant pas d'études n'est « que » de $20 \%$ alors que, inversement, $66 \%$ d'entre elles engagent des études avant ou parallèlement à la prospection. Les dirigeants deviennent alors des experts prenant une décision en toute connaissance de cause. Cela traduit à notre sens une différence d'appréciation du rôle de l'encadrement de l'entreprise.

Les entreprises plus exportatrices sont beaucoup plus ouvertes aux études que les autres entreprises. Ces dernières privilégient l'action, certainement soutenue par l'intuition des dirigeants, et une forme de prise de risques plus importante.

L'ensemble des indicateurs utilisés permet de conclure que les performances de l'entreprise sur les marchés étrangers sont meilleures lorsque le degré d'ouverture des systèmes d'information utilisés dans le cadre du développement international est plus élevé. Les entreprises les plus exportatrices semblent plus habituées à "capter» l'information et à laisser la porte ouverte aux signaux, aux repérages, même s'ils ne correspondent pas exactement à un domaine de recherche strictement délimité autour du produit et du marché actuel de l'entreprise. Les entreprises moins exportatrices se réfugient dans des systèmes plus étroits qui leur permettent de se sécuriser grâce à un cadre de recherche étroitement centré sur l'activité de l'entreprise et plus particulièrement, sur le couple produit-marché - voire peut-être sur la seule variable produit. Ces entreprises ont des difficultés à évaluer la rentabilité du traitement de l'information pléthorique qu'elles peuvent collecter ou simplement recevoir. Dès lors, où est l'intérêt de se laisser noyer? 


\subsubsection{Analyse typologique: des comportements différenciés en matière d'ouverture des systèmes d'information}

La dernière partie de l'analyse permet l'identification de trois classes, représentant respectivement :

- $44,6 \%$ du total de l'effectif pour la classe 1 ;

- $29,3 \%$ du total de l'effectif pour la classe 2;

- $26,1 \%$ du total de l'effectif pour la classe 3 .

Outre le constat de la prédominance en nombre d'entreprises de la classe 1, plusieurs remarques peuvent être faites :

1. La structure de la classification est liée au caractère plus ou moins exportateur des entreprises : la classe 1 est composée majoritairement d'entreprises moins exportatrices tandis que la classe 2 et la classe 3 comprennent une majorité d'entreprises plus exportatrices.

2. Les entreprises de la classe 1 sont caractérisées par une gestion de l'information peu ouverte :

- $85,4 \%$ d'entre elles pensent que le préformatage du classement de l'information est indispensable;

- 46,3\% pensent qu'une information utile est une information demandée;

- 70,7\% conservent peu ou pas d'information en provenance de l'extérieur;

- $70,7 \%$ ne font aucune étude avant de lancer une prospection sur le marché international ;

- $78 \%$ des entreprises de cette classe sont moins exportatrices que la moyenne.

Ce comportement paraît rigide, peu ouvert sur l'extérieur, autosuffisant et négligeant l'information spontanée externe à l'entreprise.

Les entreprises des classes 2 et 3 sont en revanche plus exportatrices que la moyenne : $81,5 \%$ des entreprises de la classe 2 et $79,2 \%$ des entreprises de la classe 3 exportent plus de $20 \%$ de leur chiffre d'affaires.

Les caractères les plus discriminants entre la classe 1 et les classes 2 et 3 sont l'évaluation du préformatage du système d'information, la notion d'information utile et le mode de consommation des études de marché, sans toutefois que les comportements soient homogènes dans les classes 2 et 3 .

Les entreprises de la classe 2 et celles de la classe 3 pensent respectivement pour $11,1 \%$ et $8,3 \%$ que l'information utile est une information demandée; de même, 
elles préfèrent, pour $77,8 \%$ et $91,7 \%$ d'entre elles, éviter les situations de prospection sans étude préalable. Ces différents caractères ne sont pas discriminants.

En revanche, d'autres comportements divergent. On constate ainsi que parmi les entreprises de la classe 2 :

- $66,7 \%$ pensent que le préformatage du classement de l'information est indispensable;

- 29,6\% conservent la majeure partie de l'information extérieure qui leur parvient et $77,8 \%$ achètent des études de marché.

Ces entreprises préfèrent une information correspondant à un besoin précis qu'elles savent énoncer. Leur procédure de choix de marché est très tournée vers l'action, elles sont seulement $48,1 \%$ à réaliser une étude avant de lancer la prospection et $14,8 \%$ à faire l'étude parallèlement au lancement de la prospection.

Sensibles et ouvertes à l'information en provenance de l'extérieur, elles préfèrent exploiter des données plus en adéquation avec les besoins précis d'une situation donnée et sont très favorables à la réalisation d'études préalables à l'action.

Les entreprises de la classe 3 agissent différemment :

- elles travaillent majoritairement $(58,3 \%)$ à partir de données rassemblées et sont plus nombreuses que dans la classe 2 à idéaliser un système de classement préstructuré $(91,7 \%)$;

- plus nombreuses à conserver beaucoup d'information extérieure $(66,7 \%)$, elles sont en revanche beaucoup moins habituées (12,5\% des cas) que la moyenne des entreprises $(18,5 \%)$ et notamment celles de la classe 2 $(48,1 \%$ des cas) à réaliser des études avant de lancer la prospection.

Cette classe d'entreprises évalue de façon positive la structuration et la préparation de la collecte de données et des systèmes d'information, même si la réalité n'est pas toujours conforme à l'idéal envisagé.

Deux types de comportements coexistent pour l'obtention de bonnes performances :

- d'une part, des entreprises (classe 3) peu tournées vers la formalisation et la structuration des systèmes d'information, très ouvertes à l'information extérieure qu'elles conservent en grande partie, la préférant peut-être à des études de marché que seulement $25 \%$ d'entre elles ont acheté. Très actives, liant études et prospection, ces entreprises sont très ouvertes et peu rigides dans leur gestion de l'information; elles exploitent leurs qualités d'entreprises de taille moyenne, très ancrées dans un système relationnel important. La constitution de leur réseau est progressive et tout à fait dissociée des problèmes éventuels à traiter à court terme. En d'autres 
termes, ces entreprises cultivent les relations avec leur environnement, dans un souci permanent de vigilance peu formalisée beaucoup plus que dans une préoccupation de court terme de résolution de problèmes;

- d'autre part, des entreprises (classe 2) faisant preuve d'une gestion ouverte mais très ordonnée, rigoureuses, capables de capter l'information extérieure mais soucieuses par ailleurs, dans le cadre de l'exploitation de l'information, d'une importante rationalité.

L'étude effectuée valide totalement l'hypothèse émise : les entreprises utilisant des systèmes d'information très ouverts sont plus performantes que la moyenne.

La nuance est à apporter sur les modalités de « mise en œuvre de l'ouverture des systèmes ». Au-delà du clivage effectué a priori sur le critère "plus ou moins exportateur que la moyenne », une analyse approfondie révèle l'existence de trois types d'entreprises dont la structure est liée à l'importance de l'activité export. Un premier groupe d'entreprises adopte des comportements peu ouverts et obtient des résultats limités sur le marché international. Les deux autres groupes identifiés parviennent à des résultats tout à fait positifs en utilisant des méthodes de gestion de l'information différentes: le premier groupe gère la collecte et le traitement de l'information de façon très rigoureuse, le deuxième groupe travaille d'une façon plus intuitive et aléatoire.

\section{Conclusion}

\section{Une approche nuancée du traitement de l'information} dans les entreprises de taille moyenne exportatrices et performantes

Le traitement de l'information comme élément de performance des entreprises exportatrices dans les PME était le point central de la problématique de recherche. Notre travail a été orienté sur la nature de la démarche informationnelle de ces entreprises et sur les qualités d'ouverture de leurs systèmes d'information. L'objectif était de comprendre « comment » ces entreprises fonctionnent et de ne pas s'arrêter au constat classique d'un sous-équipement humain, matériel ou financier.

Cette recherche présente certaines limites. Au-delà de la limite classique dans les recherches sur les PME posée par l'exploitation des données d'un échantillon de seulement 92 entreprises, une limite importante nous paraît être posée par l'utilisation d'un indicateur de performance très simple de chiffre d'affaires export par rapport au chiffre d' affaires global, et ce, malgré les nombreuses recommandations pour une approche complexe de la notion de performance des PME exportatrices. Par ailleurs, l'approche méthodologique choisie ne nous permet pas de présenter un modèle prescriptif et d'envisager des liens de causalité entre les variables de gestion de l'information et les performances export des PME exportatrices. 
Parallèlement, nous pouvons constater plusieurs apports. Deux catégories de résultats, issues de deux phases méthodologiques, ressortent de cette étude:

- l'analyse bivariée amène à l'identification de comportements différents de gestion de l'information entre les entreprises exportant moins que la moyenne de leur secteur et celles exportant plus que cette moyenne (évaluée à $20 \%$ pour le secteur industriel);

- au-delà de ce premier constat de nature très dichotomique, il est possible d'identifier des comportements différents pour des niveaux de performance équivalents et élevés. Il y a là un apport important : la nature même des structures de type PME et de leurs multiples contraintes a été utilisée pour expliquer leurs difficultés - ou leurs incompétences - à mettre en œuvre des systèmes d'information efficaces. Or, les résultats présentés mettent en évidence une situation plus nuancée. Suivant l'analyse effectuée, deux types de comportement de gestion de l'information paraissent acceptables dans les PME du secteur industriel pour développer de bons niveaux de performance sur le marché international : un comportement que nous pourrions qualifier de « traditionnel », qui privilégie la formalisation d'une politique de gestion de l'information (attribution d'un budget, structuration des contacts, mise en place de procédures, recrutement de compétences, etc.) et un comportement plus original, très adapté aux caractéristiques des PME, privilégiant les relations et les contacts informels avec l'environnement - institutionnel ou marchand - laissant par là même, place à une forme d'aléa reposant sur l'utilisation des connaissances tacites de chaque individu.

La performance internationale est donc développée par la prise en compte rigoureuse de la ressource information (ce qui n'est pas encore courant dans les structures de taille moyenne) ou par l'exploitation des qualités relationnelles de la PME pour capter l'information et permettre l'anticipation dans un univers qualifié d'incertain où le repérage des problèmes et des décisions à prendre devient primordial.

Seules les entreprises qui n'ont pas encore perçu l'importance d'un traitement complexe de l'information dans le cadre d'une politique marketing, intégrant de très nombreuses données pour répondre à des niveaux de besoins très différents, accusent des faiblesses en matière de développement international.

La considération des spécificités des entreprises de taille moyenne permet de dénouer le paradoxe informationnel évoqué en introduction. L'approche organisationnelle est complémentaire à l'approche technologique : il s'agit de développer la capacité à capter les signaux faibles pour augmenter la connaissance autant que l'aptitude à maîtriser les moyens technologiques qui serviront de supports à une information très formalisée. 
L'évaluation des systèmes d'information des PME devra tenir compte de ces caractéristiques de fonctionnement. Le développement de nouveaux outils, l'adhésion à de nouvelles bases de données renforceront l'efficience de ces organisations parce qu'elles pourront capter l'information. Mais il ne faut pas négliger le rôle des comportements propres aux entreprises de taille moyenne dans le développement de leur efficacité. Si formalisation il y a, il serait souhaitable que ce soit plutôt une formalisation de tous les savoirs individuels détenus par ces entreprises informelles qu'une formalisation trop simpliste des flux d'information explicites et facilement codifiables.

L'appui nécessaire devient alors plus un appui à la gestion des savoirs et au développement des réseaux, double condition de développement de nouvelles performances internationales.

\section{Bibliographie}

AABY, N.-E. et S.F. SLATER (1988), «Management influence on export performance : a review of the empirical literature 1978-1988», International Marketing Review, vol. 6, no 4, p. 6-24.

AlgOE (1992), Vers la compétitivité globale, PMI 90, Rapport d'enquête pour le ministère de l'Industrie, La Documentation française.

AMABILE, S. (1994), «D'une gestion substantive de l'information à une organisation procédurale de l'attention ", Sciences de la Société, no 33 , octobre.

AMMEUX, J.-P. (1984), Les PME en France : leur place dans le système productif et les exportations nationales, thèse de $3^{\mathrm{e}}$ cycle, Université Paris I, Panthéon Sorbonne.

AvisCo CONSEIL (1988), PMI et Exportation, Étude réalisée pour le ministère de l'Industrie.

BAILE, S. et I. SolE (1995), «PME et investissements en TI : nécessité d'une évaluation spécifique », dans Actes du colloque Innovation et organisation des PME, Paris, octobre.

BAUMARD, P. (1996), Les organisations déconcertées ; la gestion stratégique de la connaissance, Paris, Masson.

BESH, B. et G. GUIEU (1993), « Restructuration et stratégie des entreprises au cours des années 80 », dans Alain Noël (dir.), Perspectives et management stratégique, Paris, Economica.

BLILI, S. et L. RAYMOND (1994), «Technologies de l'information : menaces et opportunités pour la PME », dans P.-A. Julien, Pour des PME de classe mondiale, Montmagny, Transcontinentales.

BouRgEOIS, P. et L. ESTIVAL (2000), « Export : comment l'État aide les PME », Les cahiers de l'Industrie, $\mathrm{n}^{\circ}$ 57, mai.

BOUTARY, M. (1998), Le traitement de l'information comme élément de performance des entreprises exportatrices, thèse en sciences de gestion, Université de Toulouse I.

BouQuin, H. (1989), «Audit », Encyclopédie de Gestion, Paris, Economica. 
BOYER M. (1993), «La performance des PMI dans l'attaque des marchés internationaux : Application au cas des punchs et liqueurs réunionnais », Présentation au colloque PME PMI et Développement international, Aix-en-Provence, juin.

CAVUSGIL, S.T. (1981), «Organizational characteristics associated with export activity », Journal of Management Studies, vol. 21, $\mathrm{n}^{\circ} 1$.

CAVusGiL, S.T. et J. NEVIN (1981), «Internal determinants of export marketing behavior : an empirical investigation », Journal of Marketing Research, vol. 18, février.

CAVusGil, S.T. et Y.M. GodiWALLA (1982), « Decision-making for international marketing : a comparative review », Management Decision, vol. 20, $n^{\circ} 4$, p. 47.

CHAPELliER, P. (1994), Comptabilité et système d'information du dirigeant de PME; essai d'observation et d'interprétation des pratiques, thèse de doctorat, Montpellier.

CHRYssochoIDIS, G. (1992), «An alternative measure of export performance», dans Marketing for Europe, Marketing for the Future, Actes, Aarhus.

COOPER, R.G. et E.J. KLEINSCHMIDT (1985), «The impact of export strategy on export sales performance », Journal of International Business Studies, vol. 16, printemps.

Croue, C. (1993), Marketing International, Paris, De Boeck Université.

DAVIS, G.B. (1974), Conceptual Foundation, Structure and Development, New York, McGraw-Hill.

DENIS, J.-E. (1990), «Une synthèse de la recherche sur le comportement des firmes à l'exportation », Document de travail 90-15, Université d'Ottawa.

DuCheneaut, B. (1996), Enquête sur les PME françaises, Paris, Laurent du Mesnil.

ERMES, GROUPE ESCP (1994), Systèmes d'information : la perspective du management, Paris, Masson.

EVRARD, Y. et F. LE MAIRE (1993), «Études et recherches en marketing », MARKET, Nathan, p. 540.

FENWICK, I et L. AMINE (1979), «Export performance and export policy : evidence from UK clothing industry », Journal of the Research Society, vol. 30, $\mathrm{n}^{\circ} 8$.

GALE, G.P. (1992), «Marketing information : an empirical analysis », Marketing for Europe, Marketing for the Future, Actes, Aarhus, mai.

GEMUNDEN, H.G. (1991), « Success factors of export marketing : a meta-analytic critique of the empirical studies », New Perspectives on International Marketing, New York, Stanley J. Paliwoda.

HERRMANN, J.-L. (1998), Le Marketing en PME manufacturières : les pratiques de quelques entreprises lorraines lors du développement de produit, Actes du IV Congrès francophone sur la PME, Nancy.

HUNT, C. et V. ZARTARIAN (1990), Le renseignement stratégique au service de votre entreprise, Paris, First.

JoYAL, A. (1993), «Les PME exportatrices : caractéristiques et comportements », dans Actes du colloque PMI et Développement international, Aix-en-Provence.

JULIEN, P.-A. (1993), « Small business as a research subject : some reflexions on knowledge of small business and its effects on economy theory », Small Business Economics, vol. 5 , p. $157-166$. 
JULIEN, P.-A., dir. (1994), PME, Bilan et perspectives, Paris, Economica.

Julien, P.-A., J.-M. Toulouse, C. Ramangalahy et M. Morin (1998), Comportements d'information, compétitivité et performance des $P M E$ exportatrices, IV ${ }^{\mathrm{e}}$ Congrès francophone de la PME, Metz, octobre.

KASHANI, K. (1995), « Marketing futures : priorities for a turbulent environment », Long Range Planning, vol. 28, n $^{\circ}$, p. 87-98.

LE MoIGNE, J.-L. (1986), « Vers un système d'information organisationnel », Revue Française de Gestion, novembre.

LE VIGOUREUX, F. (1995), Essai de définition de la moyenne entreprise, Cahier 1/95, IAE Basse-Normandie.

LESCA, H. (1992), «Information stratégique et surveillance de l'environnement de l'entreprise », Encyclopédie du management, tome 1, Paris, Vuibert, p. 846-856.

MADHOK, A. (1996), «Local markets and global education: the firm in the international knowledge economy », Management International, vol. $1, \mathrm{n}^{\circ} 1$.

MATTEI PASTURE, F. DE (1993), «L'information, élément immatériel à gérer et à maîtriser par la PME », Document de travail, Université de Belgique, Mons Hainaut.

MAYERE, A. (1995), « La gestion des savoirs face au nouveau modèle industriel », Revue Française de Gestion, octobre, p. 8-16.

MONNOYER, M.-C. (1993), «La fonction d'information pour l'exportation», dans Actes du colloque PME PMI et Développement international, Aix-en-Provence.

MONNOYER, M.-C. (1995), « Le système d'information de gestion : du concept à l'opératoire », Présentation au colloque international «Économie de l'information », ENSSIB CERSI, Lyon, mai.

NoNAKA, I. (1990), «Redundant, overlapping organization: a Japanese approach to managing the innovation process », California Management Review, printemps.

NONAKA, I. (1994), «A dynamic theory of organizational knowledge creation », Organization Science, vol. $5, \mathrm{n}^{\circ} 1$, février.

RAYMOND, L. «Management information systems : problems and opportunities », International Small Business Journal, vol. 7, $\mathrm{n}^{\circ} 4$.

RAYMOND, L. (1984), «Personal and attitudinal correlates of MIS success in small business », Journal of Business Administration, vol. 15.

RAYMOND, L. (1990), "Organizational context and information systems success : a contingency approach », Journal of Management Information Systems, vol. 6, $\mathrm{n}^{\circ} 4$, printemps.

REID, S. (1984), «Information acquisition and export entry decisions in small firms », Journal of Business Research, New York, juin, vol. 12, nº 2, p. 141.

Roncin, A. (1982), «L'engagement des PMI dans l'exportation », Économie et Statistiques, $\mathrm{n}^{\circ} 148$, p. 49-79.

Roux, E. (1991), Les facteurs explicatifs de la décision d'exporter en PMI : rôle de l'attitude du dirigeant envers le risque, thèse d'État, IAE Aix-en-Provence.

SAPORTA, B. (1986), Stratégies pour la PME, Paris, Montchrestien.

USUNIER, J.-C. (1992), Commerce entre cultures, Paris, Presses universitaires de France, coll. « Gestion ». 